ARTICLE OPEN

\title{
Engineered lactobacilli display anti-biofilm and growth suppressing activities against Pseudomonas aeruginosa
}

\author{
Todd C. Chappell ${ }^{1}$ and Nikhil U. Nair $\mathbb{D}^{1 凶}$
}

Biofilms are an emerging target for new therapeutics in the effort to address the continued increase in resistance and tolerance to traditional antimicrobials. In particular, the distinct nature of the biofilm growth state often means that traditional antimcirobials, developed to combat planktonic cells, are ineffective. Biofilm treatments are designed to both reduce pathogen load at an infection site and decrease the development of resistance by rendering the embedded organisms more susceptible to treatment at lower antimicrobial concentrations. In this work, we developed a new antimicrobial treatment modality using engineered lactic acid bacteria (LAB). We first characterized the natural capacity of two lactobacilli, $L$. plantarum and $L$. rhamnosus, to inhibit $P$. aeruginosa growth, biofilm formation, and biofilm viability, which we found to be dependent upon the low $\mathrm{pH}$ generated during culture of the LAB. We further engineered these LAB to secrete enzymes known to degrade $P$. aeruginosa biofilms and show that our best performing engineered $L A B$, secreting a pathogen-derived enzyme (Pel $\left.A_{h}\right)$, degrades up to $85 \%$ of $P$. aeruginosa biofilm.

npj Biofilms and Microbiomes (2020)6:48; https://doi.org/10.1038/s41522-020-00156-6

\section{INTRODUCTION}

As an important virulence factor for pathogenic microbes, biofilms are associated with an expanding array of pathologies, including various airway, gastrointestinal, and ocular infections, endocarditis, periodontitis, osteomyelitis, cystitis, and chronic wounds ${ }^{1-7}$. Biofilms represent a distinct growth state, morphologically distinguished by bacteria residing within a self-produced matrix of extracellular polymeric substances (EPS), that may include proteins, extracellular DNA (eDNA), polysaccharides, and lipids ${ }^{8}$. Within the biofilm, isogenic cells exhibit phenotypic diversity that is driven by the discrete microenvironments created by metabolite, ion, gas, and antimicrobial gradients within the biofilm. Biomedically, this phenotypic diversity manifests as distinct tolerances or resistances to traditional antimicrobials as well as the host immune system ${ }^{9,10}$. Additionally, biofilms stabilize surface colonization and are frequently less susceptible to traditional methods of surface decontamination, exacerbating the recalcitrance to treatment. Thus, clearance of mature biofilms is an essential component for the successful resolution of numerous infections, especially those that are chronic or recurrent in nature.

Invasive burn wounds and chronic wounds, or wounds that fail to progress through the later stages of the normal healing process, are commonly contaminated or colonized by a multitude of biofilm-forming organisms. Standard treatments for these wound types include nanocrystalline silver, silver sulphadiazine, iodine, or topical antibiotics. However, these treatments are often ineffective at reducing wound infection, add unnecessary expense, and/or inhibit the healing process ${ }^{11-14}$. Further, extensive use of these treatments has bred a large population of multidrug-resistant microbes for which new treatments that target both planktonic and biofilm cells are necessary.

A widespread biofilm targeting strategy is the enzymatic degradation of biofilm polymer(s) to decrease surface adhesion and return the entrained bacteria to a more treatable phenotype ${ }^{15-21}$. Rapid advancement in synthetic biology and probiotic therapies have led to interest in developing engineered bacteriotherapies or live biotherapeutic products. These "smart", bacteria-based therapeutic delivery vectors provide sustained delivery of the therapeutic and dynamically respond to environmental signals, while retaining their innate probiotic qualities ${ }^{22-25}$. Recent examples of bacteriotherapies include the delivery of enzymes, antimicrobials, metabolites, or anti-inflammatory proteins to combat metabolic deficiencies, tumors, inflammation, biofilms, and infections ${ }^{22,26-32}$. In this study, we construct and assess the utility of genetically engineered probiotic bacteria as anti-biofilm and antimicrobial agents against the common wound pathogen Pseudomonas aeruginosa.

We selected lactic acid bacteria (LAB) as the chassis strains for the bacteriotherapy due to their broad-spectrum antimicrobial and wound healing capacities, genetic tractability, and wellcharacterized expression systems for the production and secretion of heterologous proteins. Furthermore, Several LAB have been shown to impair the growth of drug-resistant $P$. aeruginosa clinical isolates ${ }^{33}$. More specifically, Lactobacillus plantarum and Lactobacillus rhamnosus, the species used in this work, enhance the outcome of mouse $P$. aeruginosa infection models, increase epithelial migration, and are equally as effective as current treatments when applied to human burn wounds ${ }^{34-37}$. We add to this body of evidence, showing that $L$. plantarum WCFS1 and $L$. rhamnosus GG (LGG) are effective inhibitors of planktonic growth, biofilm formation, and the viability of biofilm-embedded cells (biofilm viability) of the burn wound isolate $P$. aeruginosa PA14 (PA14). We further increase the usefulness of $L$. plantarum and LGG by engineering them to secrete enzymes known to degrade PA14 biofilms and demonstrate the efficacy of this design for degradation of mature PA14 biofilms.

\section{RESULTS}

L. plantarum and LGG inhibit PA14 growth in a pH-dependent manner

The feasibility of $L$. plantarum and LGG as therapeutic vectors was first analyzed by characterizing their innate capacity to inhibit PA14 growth using an agar-well diffusion assay and dilutions of

${ }^{1}$ Department of Chemical \& Biological Engineering, Tufts University, Medford, MA, USA. ${ }^{\circledR}$ email: nikhil.nair@tufts.edu 
a

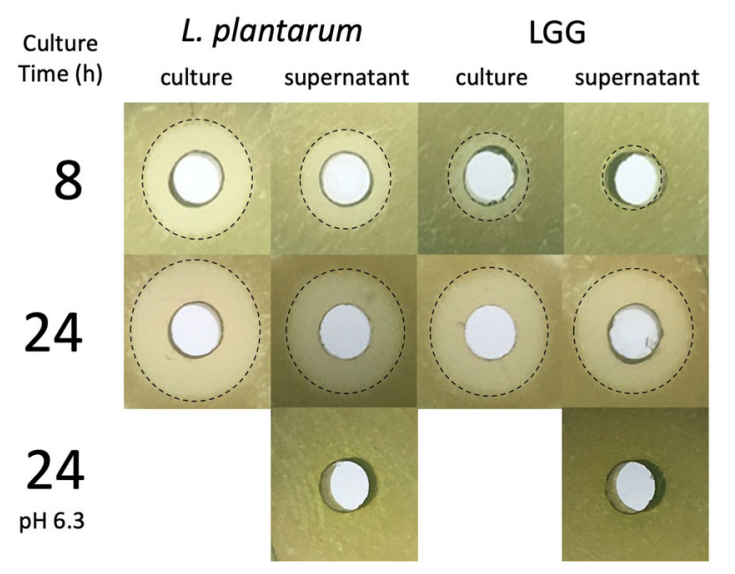

b
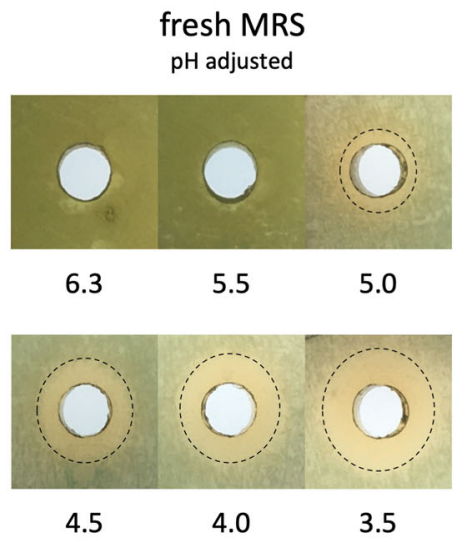

C

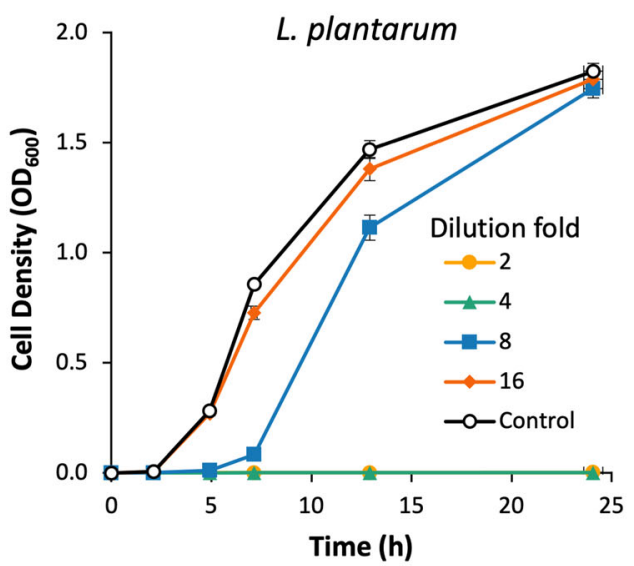

d

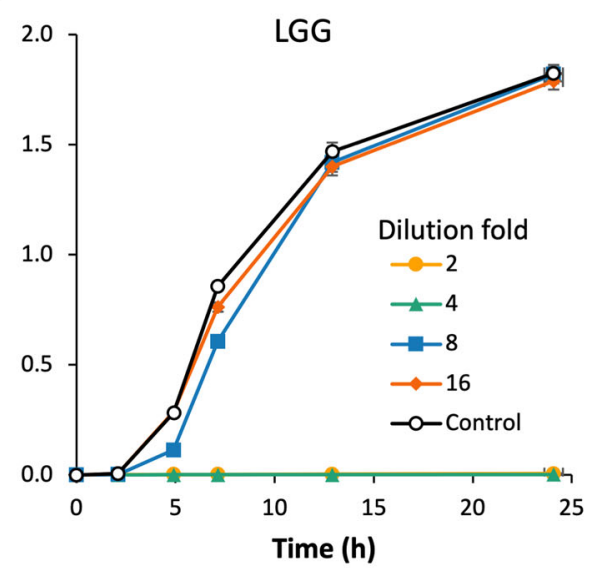

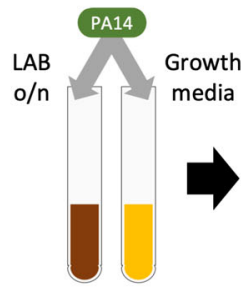

Innoculate

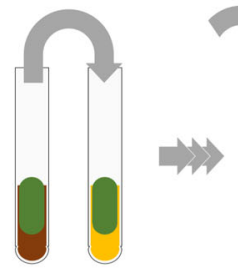

Serially dilute

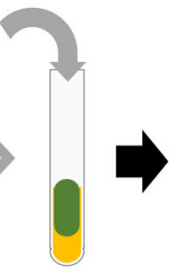

Fig. 1 Inhibition of PA14 growth by LAB. a Agar well diffusion assay of L. plantarum and LGG cultures and supernatants grown in MRS medium. Culture time was either 8 or $24 \mathrm{~h}$. $\mathrm{pH}$ adjustment abrogates inhibitor activity of supernatants. b Agar well diffusion assay of pHadjusted fresh MRS medium. The $\mathrm{pH}$ of the acid-adjusted medium is located below the plate image to which it refers. Cultures of planktonic PA14 with c L. plantarum and d LGG supernatants show inhibition at low dilution factors. $n=9$ from three separate experiments for all conditions. Error bars represent \pm 1 SEM. Workflow for $\mathbf{c}$ and $\mathbf{d}$ is shown: The LAB supernatants were inoculated with PA14, which were serially diluted into fresh PA14 cultures. Each line represents a different dilution factor of the LAB supernatant.

LAB cultures in a modified MIC assay. The agar-well diffusion assay was used to determine the aeration and duration of LAB culture that maximally inhibited PA14 growth. When L. plantarum cultures were grown aerobically in a test tube or flask, growth inhibition of PA14 moderately increased (Supplementary Fig. 1). However, culture aeration had no impact on LGG growth inhibition of PA14. Early phase L. plantarum and LGG cultures (grown $\leq 4 \mathrm{~h}$ ) and supernatants failed to inhibit PA14 growth, while late-stage ( $\geq 8 \mathrm{~h}$ ) cultures and supernatants of both organisms inhibited PA14 growth (Fig. 1a, Supplementary Fig. 1). The $24 \mathrm{~h}$ cultures of $L$. plantarum were marginally more inhibitory than those of LGG; yet the supernatants of both LAB exhibited similar growth inhibition against PA14. Generally, we found that PA14 growth inhibition increased with $L A B$ culture duration and cultures were more inhibitory than cell-free supernatants. The $\mathrm{pH}$ of $24 \mathrm{~h}$ supernatants was 3.8-3.9 and when we adjusted their $\mathrm{pH}$ back to the starting $\mathrm{pH}$ of 6.3, we observed no growth inhibition. To determine if the inhibitory activity was due to $\mathrm{pH}$ alone or a factor that was active at low $\mathrm{pH}$, we adjusted the $\mathrm{pH}$ of fresh MRS down to that of spent media and evaluated its inhibitory activity against PA14. Decreasing the medium $\mathrm{pH}$ increased growth inhibition and when adjusted to $\mathrm{pH} 3.5$, the medium had similar inhibitory activity as that of a $24 \mathrm{~h}$ LAB culture of $\mathrm{pH}$ 3.8-3.9.

We also used a modified minimum inhibitory concentration (MIC) assay to more quantitatively evaluate the inhibition of PA14 growth by $L$. plantarum and LGG supernatants over time and 
a

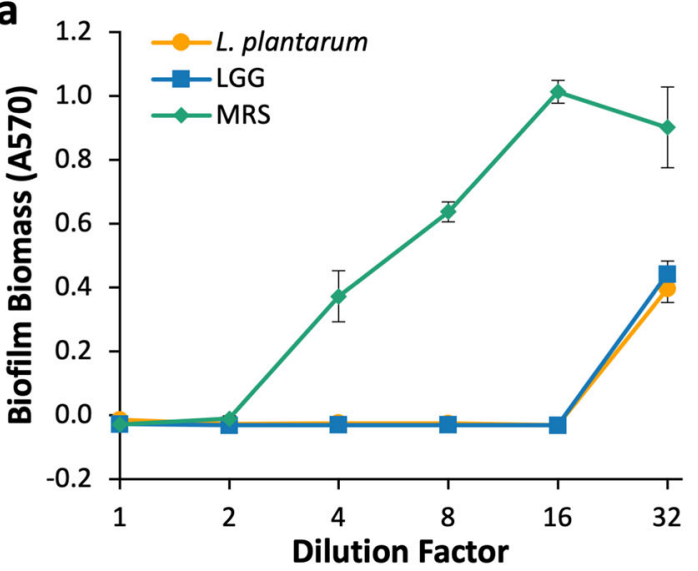

b
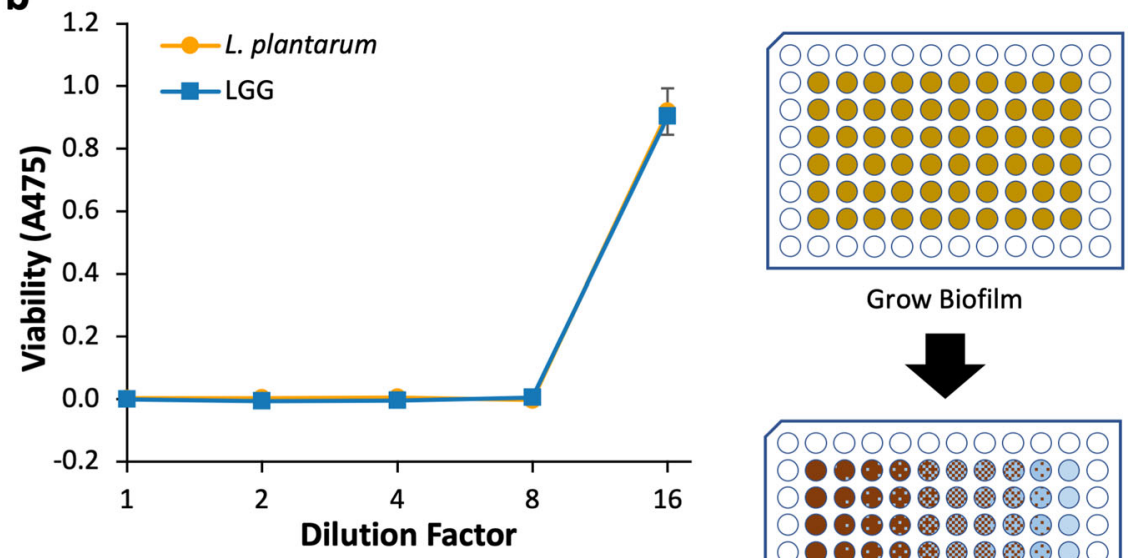

Grow Biofilm

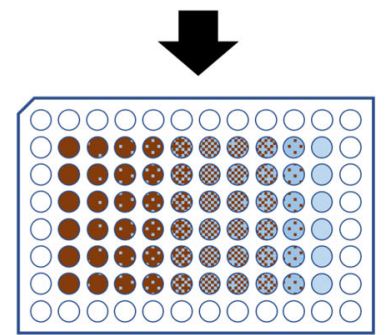

C

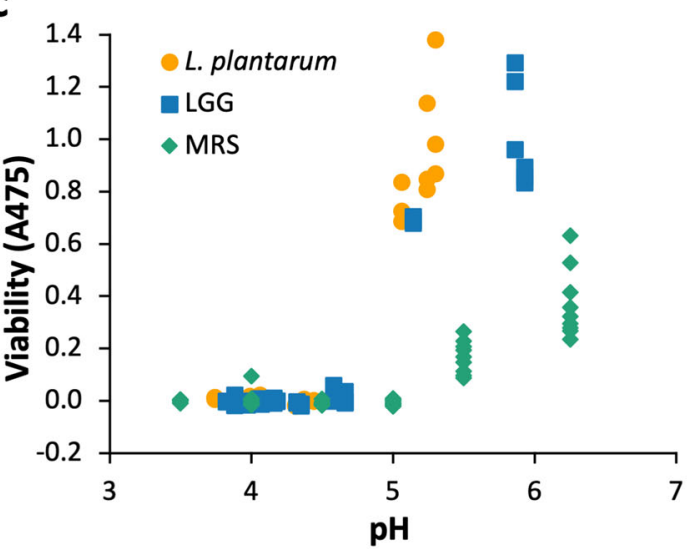

Incubate w/ LAB supernatant

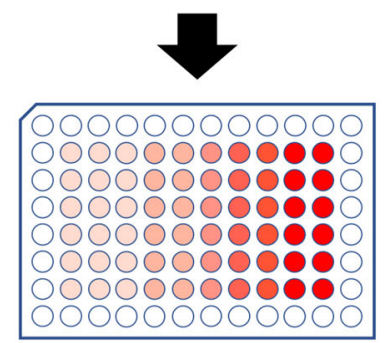

XTT viability

Fig. 2 Anti-PA14 biofilm activity of LAB. a Inhibition of PA14 biofilm formation by L. plantarum and LGG. Workflow for a was similar to that shown in Fig. 1. b Inhibition of viability of mature PA14 biofilms by L. plantarum and LGG determined by measuring XTT reduction (absorbance at $475 \mathrm{~nm}$ ). LAB cultures were diluted in PBS. c Inhibition of PA14 biofilm viability by L. plantarum and LGG compared to pH of culture diluted in PBS or MRS adjusted to a specific pH. Workflow for $\mathbf{b}$ and $\mathbf{c}$ is shown. $n=9$ from three separate experiments for all conditions. Error bars represent \pm 1 SEM.

determine the relative quantity of spent supernatant necessary for bioactivity. Supernatants from $24 \mathrm{~h}$ cultures of L. plantarum and LGG diluted up to $25 \%$ (i.e., $4 \times$ dilution) of the culture volume completely inhibited PA14 growth (Fig. 1c, d). L. plantarum supernatant diluted $8 \times$ still retained some inhibitory activity, while $8 \times$ dilution of LGG supernatants had no inhibitory activity relative to growth medium alone. A dilution of $16 \times$, or greater, of either LAB culture supernatant failed to inhibit PA14 growth.
L. plantarum and LGG inhibit PA14 biofilm formation and viability Having characterized $L$. plantarum and LGG inhibition of planktonic PA14 cells, we also analyzed the impact L. plantarum and LGG supernatants had on PA14 biofilm formation and biofilm viability (i.e., viability of cells in the biofilm matrix). We used the modified MIC assay workflow to evaluate the inhibition of PA14 biofilm formation. The supernatants from L. plantarum and LGG cultures inhibited PA14 biofilm formation in a concentration- 
a
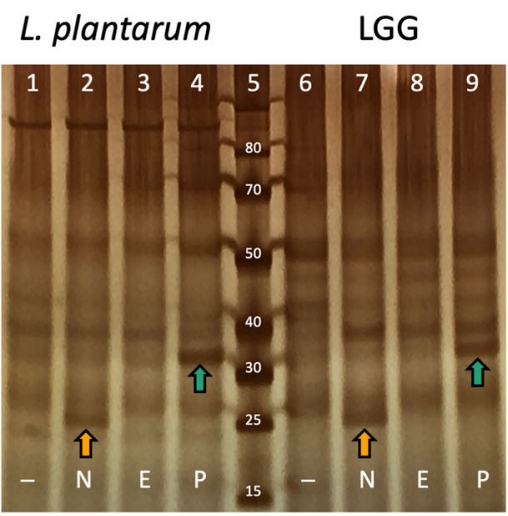

b

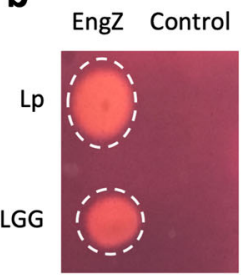

C

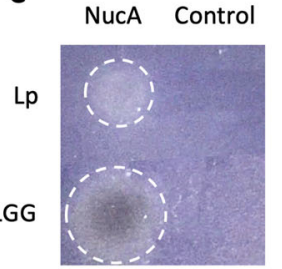

d

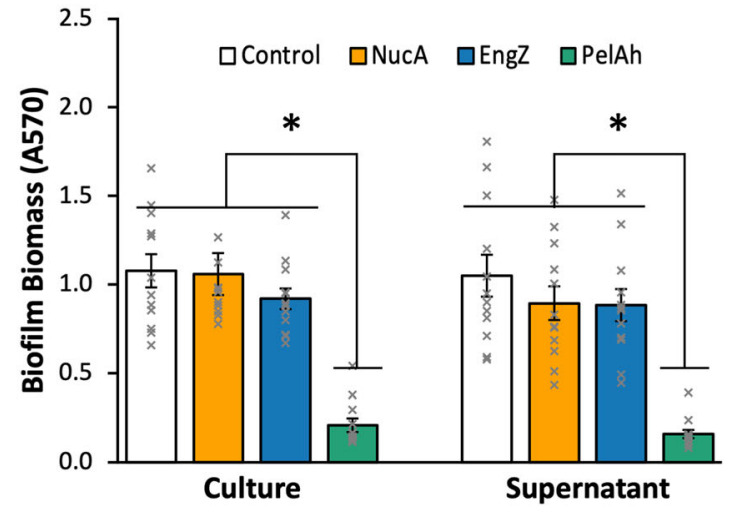

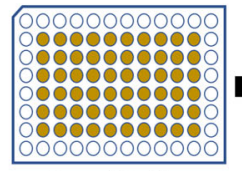

Grow Biofilm

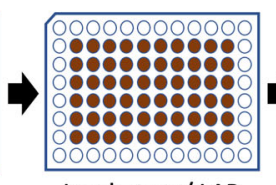

Incubate w/ LAB supernatant

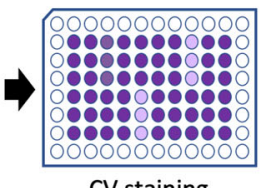

CV staining

Fig. 3 LAB-secreted enzymes degrade PA14 biofilm. a Silver stained SDS-PAGE gel of supernatants from induced LAB cultures; Lanes $1-4 L$. plantarum containing control (empty vector pTCC210,-), NucA (N), EngZ (E), and Pel $A_{h}(P)$ plasmids; Lane 5 Ladder; Lanes $6-9$ LGG containing control $(-)$, NucA $(N)$, EngZ $(E)$, and PelA $A_{h}(P)$ plasmids. Arrows denote bands for NucA $(\sim 19 \mathrm{kDa})$ and PelA $A_{h}(\sim 31 \mathrm{kDa})$. b CMCase plate assay of EngZ-expressing L. plantarum and LGG. c DNase plate assay of NucA expressing L. plantarum and LGG. d Degradation of PA14 biofilms with the cultures and supernatants of $L$. plantarum containing Control, NucA, EngZ, and Pel $A_{h}$ expression plasmids. L. plantarum culture pH following induction was $5.0 \pm 0.0,5.1 \pm 0.1,5.3 \pm 0.1$, and $5.3 \pm 0.1$ for Control, NucA, EngZ, and Pel $A_{h}$, respectively. $n=12$ from four separate experiments for each condition. * Denotes significant difference as determined by one-way ANOVA $(a=0.05)$ and Tukey HSD comparing samples of same type (e.g. cultures or supernatants); $p<0.01$. Error bars represent and pH error are \pm 1 SEM. $n=12$ for all conditions from four biological replicates. Workflow of $\mathbf{d}$ is given below histogram.

dependent manner (Fig. 2a). Only at dilutions greater than $16 \times$ did biofilm form at detectable levels. The MRS media control also inhibited PA14 biofilm formation, but only when undiluted or diluted 2-fold.

LAB cell-free supernatants also inhibited the viability of PA14 cells embedded within biofilms, as assessed by XTT dye assay ${ }^{38}$ (Fig. 2b). L. plantarum and LGG supernatants diluted by $8 \times$ or less were able to inhibit PA14 biofilm viability such that no viable cells could be detected relative to the control. When we plotted the viability against the $\mathrm{pH}$ of LAB culture dilutions, we found that the transition to viable biofilms correlates with the increase in $\mathrm{pH}$ caused by dilution into PBS (Fig. 2c). Fresh MRS buffered to $\mathrm{pH} 5$ and below completely inhibited biofilm viability-a finding in agreement with our previous finding that this medium has an innate capacity to inhibit PA14 growth when adjusted to a lower pH (Fig. 1b). Interestingly, MRS adjusted to pH 6.25 and 5.5 also inhibited PA14 biofilm viability more than the diluted LAB cultures of a similar $\mathrm{pH}$, indicating innate anti-PA14 biofilm activity in the MRS. However, we found the major driver of decreased biofilm viability to be low $\mathrm{pH}$. Generally, PA14 biofilms treated with solutions with a $\mathrm{pH} \leq 4.5$ were nonviable, while solutions with a $\mathrm{pH} \geq 5.2$ were viable. We also found that for a given $\mathrm{pH}$, undiluted spent $L A B$ supernatant is more inhibitory compared to that diluted in PBS.

L. plantarum secreted matrix-degrading enzymes disrupt mature PA14 biofilms

$P$. aeruginosa biofilms are predominantly composed of an array of polysaccharides (Alg or alginate, Psl, Pel), and eDNA, and the specific composition is dependent upon the genetic background and environment ${ }^{39}$. The strain P. aeruginosa PA14, a burn wound isolate, does not contain a functional operon to produce Psl, and does not produce alginate as a biofilm component ${ }^{40,41}$. Instead, PA14 produces biofilms predominantly composed of Pel polysaccharide and eDNA ${ }^{42}$. P. aeruginosa biofilms containing these components were previously shown to be sensitive to enzymatic degradation by solutions containing DNase, cellulase, or native glycoside hydrolases produced by $P$. aeruginosa to release biofilm cells and transition to planktonic growth ${ }^{16,43,44}$. We constructed broad host range $L A B$ expression vectors for secretion of the cellulase EngZ, a processive endoglucanse from the Gram-positive Clostridium cellulovorans; NucA, a thermostable nuclease from Staphylococcus aureus, a pathogen found to infect similar sites as $P$. aeruginosa; and $\mathrm{Pel}_{\mathrm{h}}$ (the hydrolase domain of PelA) a native glycoside hydrolase from $P$. aeruginosa that hydrolyzes the Pel polysaccharide.

We validated the expression and secretion of the biofilm degrading enzymes from $L$. plantarum and LGG using SDS-PAGE of induced culture supernatants and enzyme activity assays. LAB cultures that contained NucA, EngZ, or Pel $A_{h}$ expression vectors had protein bands and/or enzymatic activity in the filtered supernatants, which indicates successful secretion of the intended enzymes. Specifically, the supernatants of NucA-expressing and Pel $A_{h}$-expressing $L A B$ contained protein bands of the appropriate size (Fig. 3a) but we saw no visible band for EngZ. The larger molecular weight of EngZ compared to NucA and Pel $A_{h}$ puts it in a region where numerous other protein bands in the gel make it difficult to resolve individual proteins, so we also checked for enzymatic activity. We confirmed that the supernatants of LAB secreting EngZ had CMCase activity (Fig. 3b), whereas the supernatants of LAB secreting NucA had DNase activity (Fig. 3c).

We tested the ability of $L A B$ cultures expressing and secreting NucA, EngZ, or PelA $A_{h}$ as well as their cell-free (filtered) supernatants, to degrade mature PA14 biofilms (Fig. 3d, Supplementary Fig. 2). We chose to induce the cultures in $\mathrm{BHI}$ to decouple the 
a

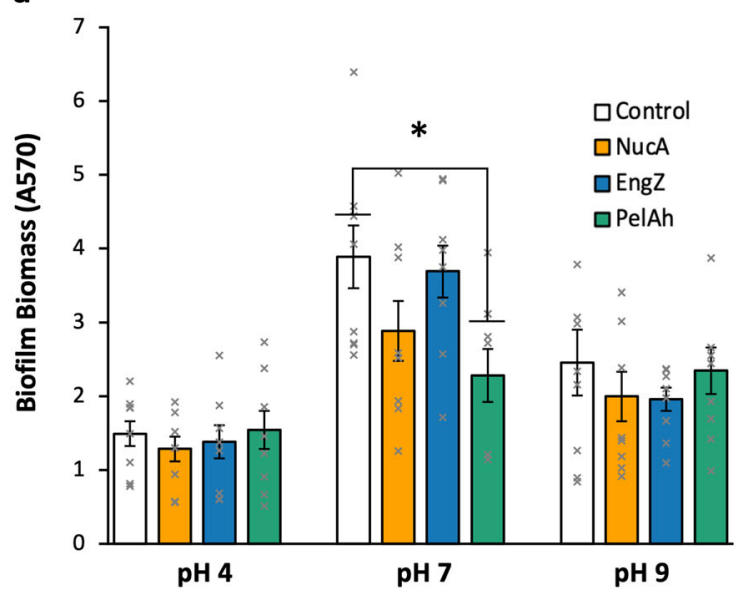

b (i)

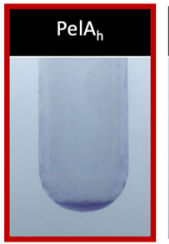

(iii)

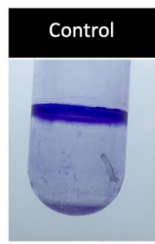

(ii)

PelA $\mathrm{pH} 7$

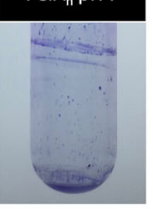

(iv)

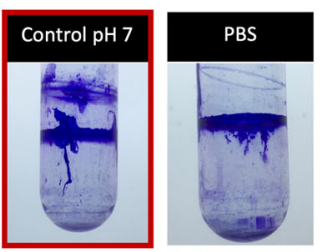

Fig. 4 Effect of enzyme secreting L. plantarum culture supernatants on PA14 biofilm. a Degradation of PA14 biofilms with supernatants of L. plantarum containing control, NucA, EngZ, and PelA $\mathrm{h}_{\mathrm{h}}$ plasmids buffered to $\mathrm{pH}$ 4.0, $\mathrm{pH}$ 7.0, and pH 9.0. Workflow is the same given in Fig. 3. * Denotes significant difference as determined by one-way ANOVA $(a=0.05)$ and Tukey HSD comparing samples of same pH; $p<0.05$. Error bars represent \pm 1 SEM. $n=9$ from three separate experiments for each condition. b Qualitative analysis of PA14 biofilms formed in culture tubes and treated with Pel $A_{h}$ expressing $L$. plantarum supernatant with $\mathrm{pH}$ (i) unadjusted and (ii) adjusted to 7.0; supernatant from L. plantarum containing empty vector with $\mathrm{pH}$ (iii) unadjusted and (iv) adjusted to 7.0; (v) PBS.

growth and inhibition of biofilm formation that we previously characterized in MRS, from the biofilm degradation capacity of the secreted enzymes. Cultures and supernatants of $L$. plantarum expressing Pel $A_{h}$ were highly effective at biofilm degradation, resulting in $80 \%$ and $85 \%$ reduction in biofilm biomass, respectively (Fig. $3 \mathrm{~d}$ ). L. plantarum Pel $\mathrm{A}_{\mathrm{h}}$ supernatants maintained similar percent biofilm degradation even at 8-fold dilution (Supplementary Fig. 3). However, EngZ and NucA expressing cultures and supernatants were ineffective at degrading PA14 biofilms. When we applied induced LGG cultures expressing PelA $A_{h}$ to PA14 biofilms, there was a considerable increase in biofilm biomass, even when the broad-spectrum Gram-negative antimicrobial polymyxin B was added to the culture to selectively inhibit additional PA14 growth (Supplementary Fig. 2). We observed less biofilm when LGG supernatants were added to PA14 biofilms. Much of the biofilm was evident on the bottom of the well when LGG cultures were added to PA14 biofilms, but not with LGG supernatants or untreated PA14 biofilms. Though LGG $\mathrm{Pel}_{h}$ supernatants did degrade PA14 biofilms, they required polymyxin $B$ to inhibit PA14 for noticeable degradation relative to PA14 biofilms incubated with $L$. plantarum supernatants containing the empty vector control. No biofilm was present when LGG was cultured in wells that did not contain PA14 biofilms, suggesting that PA14 may aid in LGG surface adhesion.

Culture $\mathrm{pH}$ determines effectiveness of engineered L. plantarum anti-biofilm activity

Having established the significance of $\mathrm{pH}$ for PA14 growth and viability when treated with $L A B$ cultures and supernatants, and knowing the optimal $\mathrm{pH}$ for NucA and EngZ are $9-10$ and $\sim 7^{45,46}$, respectively, we postulated that we could enhance NucAmediated and EngZ-mediated biofilm degradation by modulating the $\mathrm{pH}$-induced LAB supernatants. However, increasing culture $\mathrm{pH}$ did not significantly enhance biofilm degradation by NucA or EngZ relative to the control (Fig. 4a). We found no biofilm degradation by any of the enzymes when supernatants were buffered to $\mathrm{pH} 4.0$ or $\mathrm{pH}$ 9.0. Interestingly, but unsurprisingly, formation of biofilm biomass was dramatically enhanced at $\mathrm{pH} 7.0$ for all supernatants, although PelA $A_{h}$ was still effective at lowering biofilm biomass by $40 \%$ relative to the control. NucA also moderately decreased PA14 biofilm biomass at pH 7.0, but the difference was not found to be significant $(p>0.05)$.
While performing the biofilm degradation assay, it became apparent that the large increase in crystal violet (CV) staining at $\mathrm{pH}$ 7.0 and 9.0 was due to formation of additional PA14 biofilm. We illustrated this additional biofilm formation in test tubes (Fig. 4b). Mature biofilm treated with empty vector supernatant had a single biofilm at the air-solid-liquid interface at the original height of the culture volume during biofilm formation. When the control supernatant solution was buffered to $\mathrm{pH} 7.0$, and added to the mature biofilm, a second biofilm formed at the height of the control supernatant, accounting for the higher biofilm biomass detected in the microplate assay. This second biofilm was not present when the mature biofilm was incubated with PBS, which reveals that PA14 can utilize residual nutrients in the $L$. plantarum supernatant to form additional biofilm. The unmodified and $\mathrm{pH}$ 7.0 buffered Pel $A_{h}$ supernatants degraded the mature biofilm, however, some minimal new biofilm was formed when the $\mathrm{pH}$ was adjusted to $\mathrm{pH} 7.0$.

\section{DISCUSSION}

$L A B$ effect their antimicrobial activity though a variety of mechanisms, including the production of antimicrobial proteins/ peptides, inhibitory metabolites, and organic acids. While $L$. plantarum WCFS1 produces three bacteriocins (plantaricins A, EF, and $\mathrm{JK}$ ), all of which act against a relatively narrow range of physiologically similar Gram-positive bacteria ${ }^{47,48}$, and L. rhamnosus GG produces an array of antimicrobial peptides, with varying degrees of activity against both Gram-positive and Gram-negative bacteria ${ }^{49}$, we found the distinguishing inhibitory factor of LAB supernatants and cultures against PA14 growth to be low $\mathrm{pH}$. The inhibitory activity of both $L$. plantarum and LGG inversely correlated to decreasing $\mathrm{pH}$ and was abolished if the LAB supernatant was buffered to a more neutral $\mathrm{pH}$. As heterofermentors, we expect both lactobacilli to produce lactic acid and acetic acid as fermentation products ${ }^{50,51}$. Though both of these acids inhibit the growth of Gram-negative pathogens like $P$. aeruginos $a^{52-54}$, PA14 growth inhibtion was indistinguishable from growth medium buffered to an equivalent $\mathrm{pH}$ range, indicating that the identity of the acid was not very important. The low $\mathrm{pH}$ of the LAB supernatants was also important for decreasing biofilm formation and biofilm viability, and was a major factor in the success of the degradation of biofilm by engineered LAB. 
Buffering the supernatants of $L A B$ cultures directly or by dilution in PBS to more neutral $\mathrm{pH}$ resulted in maintenance of biofilm viability and the capacity to form new or more biofilms. Indeed, other investigations into multispecies bacterial communities have revealed that $\mathrm{pH}$ is a major determinant of success and failure within the community, and can generate a competitive advantage that results in elimination of acid-intolerant species ${ }^{55}$.

As a topical wound therapy, maintenance of the low $\mathrm{pH}$ would be beneficial for pathogen load reduction and clearance. Dilute organic acid solutions (e.g. acetic acid) have been used as a firstaid measure to stave off infection, yet we found that LAB cultures were more inhibitory than their acidic supernatants alone. Valdez et al. also found that $L$. plantarum cultures are more inhibitory to $P$. aeruginosa than the supernatants alone ${ }^{56}$. L. plantarum is known to remain viable at $\mathrm{pH}$ values below those found following $24 \mathrm{~h}$ culture in MRS $(\mathrm{pH}<4)$, which would explain why cultures are better at inhibiting PA14 compared to supernatants; L. plantarum can continue to acidify, and increase inhibitory activity, by utilizing residual nutrients in the media or from the LB agar plates onto which they were applied. This illustrates a major advantage to the use of $L A B$ cultures over acidified solutions or supernatants. $L A B$ cultures can continue to acidify their environment when given additional nutrients, thus maintaining an antimicrobial environment. Similarly, LAB cultures could conceivably continue to consume fermentable substrates in the wound bed, competing with pathogens for nutrients, and counteracting the return to neutral $\mathrm{pH}$ by the natural buffering capacity of the blood ${ }^{57}$. In fact, the skin normally maintains an acidic $\mathrm{pH}(4.0-5.5)$, and the normal wound healing processes-including decreased metalloprotease activity, oxygenation, epithelial migration, and angiogenesis-are correlated with acidic $\mathrm{pH}^{58,59}$. Conversely, elevated wound $\mathrm{pH}$ is often associated with chronic wounds ${ }^{57}$. Thus, LAB-mediated acidification should create an inhospitable environment for acidintolerant pathogens and is not expected to have a negative impact on the normal wound healing process.

Interestingly, we found that even though the two lactobacilli investigated in this study $-L$. plantarum and LGG_inhibited PA14 viability by lowering the culture $\mathrm{pH}$, their outcomes were divergent when applied to biofilms. Though $L$. plantarum secreting Pel $A_{h}$ degraded PA14 biofilms, LGG secreting Pel $A_{h}$ increased biofilm biomass, which we found to be the result of two factors. First, the considerable increase in biofilm biomass seen on the bottom of the culture well following the addition of LGG culture to PA14 biofilms (Supplementary Fig. 2) was dependent upon the presence of LGG culture and an extant PA14 biofilm matrix, suggesting LGG adheres to PA14 cells or EPS. While LGG is known to produce adherent biofilms, it is not known to do this when grown on $\mathrm{MRS}^{60}$, which we found as well. Second, we found that the addition of LGG supernatants also increased biofilm biomass and resulted in a second layer of PA14 biofilm, such as that shown in Fig. 4b, unless the antimicrobial polymyxin $B$ was added to the culture. This was possible because LGG grew less well in $\mathrm{BHI}$, and the culture $\mathrm{pH}$ at the end of induction was 6.7 -7.0 , and thus the $\mathrm{pH}$ was not low enough to inhibit PA14 growth and biofilm formation. This indicates that a combined antimicrobial-LGG-PelA $A_{h}$ therapy could be effective, and it remains entirely possible that the increased adhesion of LGG could enhance its antimicrobial effect by maintaining close proximity to the pathogen-both of which may be worthy of future investigations.

After engineering the lactobacilli to secrete a series of biofilm degrading enzymes, we found that only PelA $A_{h}$ secreted by $L$. plantarum was effective at degrading PA14 biofilms. Surprisingly, DNAse and EngZ secreted by $L$. plantarum were unable to appreciably degrade PA14 biofilms even though previous investigations have shown the efficacy of enzymes of these classes to be effective anti-biofilm agents against this strain $^{43,44}$. We verified the secretion and activity of NucA and EngZ in the LAB supernatant and optimized the supernatant $\mathrm{pH}$ for the activity of these enzymes, and still found no significant benefit. The activity of these enzymes at elevated $\mathrm{pH}$ may be masked by the additional growth of PA14 biofilm at the elevated $\mathrm{pH}$ at which these enzymes are most active. However, EngZ exhibits 60\% activity even at $\mathrm{pH} 4.0$, and we still saw no impact on PA14 biofilm degradation ${ }^{45}$. Previous work has shown that cellulase extracts from Trichoderma viride or Aspergillus niger can degrade PA14 biofilms ${ }^{42,44}$. However, the biofilm degrading capacity was not attributed to any single enzyme or endoglucanase activity and the exact composition of the extract is unknown. Further, activity on Pel is likely due to substrate promiscuity, which is often enzymedependent. Thus, EngZ may not have the same range of relaxed substrate specificity as $T$. viride or $A$. niger cellulases. The differences in our observations compared to that in literature could also be due to differences is assay conditions. Specifically, PA14 biofilms degradation by DNase was shown in flow cells, where DNA is known to play an integral role in the structure of biofilm stalks at the solid-liquid interface when under flow ${ }^{42,61}$. DNA may not play the same role in static batch cultures where the biofilm forms at the air-solid-liquid interface. Additionally, the DNA present in flow cell biofilms only plays an important adhesive role in early stage attachment ${ }^{43}$, and may not play a critical role in maintenance of mature biofilms. DNA also contributes to a plethora of interesting phenotypes in the biofilm, including chelating cations, inducing antibiotic resistance, promoting inflammation, and aiding extracellular electron transport, all of which are important metrics by which to test this therapy in the future ${ }^{62-65}$.

Through the development of this bacteriotherapy for the disruption of PA14 biofilm, we learned potentially important design rules for engineered bacteriotherapies that target pathogenic biofilms. First, selection of an appropriate organism as the chassis to engineer for the bacteriotherapy is essential. Specifically, the chassis organism's ability to inhibit pathogen growth, biofilm formation, and it's impact on mature biofilms, using the assays described in this work, can determine whether the organism will act as an effective bacteriotherapy. We found that prevention of additional biofilm formation and pathogen growth is a key to the degradation process. Either the inhibitory activity of low $\mathrm{pH}$, found with $L$. plantarum cultures, or the addition of the inhibitory antibiotic polymyxin B, used with LGG cultures, was necessary for biofilm degradation. The polymyxin $B$ biosynthetic pathway has been cloned and expressed heterologously, which could provide an effective strategy for generating an inhibitory environment independent of $\mathrm{pH}$ and might be necessary for in vivo application, where the wound bed $\mathrm{pH}$ can fluctuate ${ }^{66}$. Additionally, the selection of the appropriate enzyme for biofilm degradation is equally important. We found that PA14-derived Pel $A_{h}$ was most effective at degrading PA14 biofilm, and that other enzymatic activities thought to be effective at degrading PA14 biofilms were ineffective in our assay. Frequently, genes have been identified within the genome of biofilm-forming organisms that function to degrade the biofilm and release the embedded cells. However, their expression is often suppressed during the biofilm growth phase to ensure biofilm integrity. Therefore, we propose that the enzymes for biofilm degradation should be sourced from the pathogen itself, as these native enzymes were "designed" to degrade the EPS polymers. Such observations are consistent with previous studies ${ }^{15,16}$. Still, the activity of the enzyme derived from the pathogen may not be effective under the applied conditions, as we found with the $\mathrm{pH} 4$ condition. Thus, the application must strike a balance between pathogen growth inhibition, enzyme activity, and bacteriotherapy culture/environmental $\mathrm{pH}$, which we found to be the $\mathrm{pH}$ 5.0-5.3 range of induced L. plantarum cultures (Fig. 5). The $\mathrm{pH}$ working range of the treatment could be further extended through the addition of an antimicrobial such as polymyxin $B$, which then 


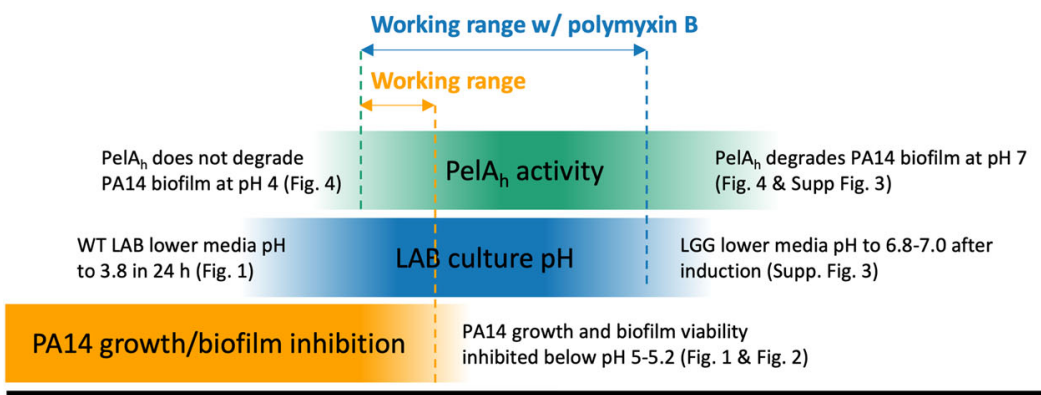

3.8

5.2
pH

Fig. 5 Degradation of PA14 biofilms by PelA $A_{h}$-secreting LAB was defined by competing pH constraints that balanced PelA $A_{h}$ activity, LABmediated acidification, and PA14 growth inhibition. This range can be extended through the addition of the polymyxin B, which inhibits PA14 growth and removes to upper pH limit set by PA14 biofilm and growth inhibition.

removes the upper $\mathrm{pH}$ constraint set by pathogen growth inhibition.

As current antimicrobial treatments decrease in efficacy, the development of novel treatments is essential for effectively treating recalcitrant infections. Traditional small molecule screening for antimicrobials has all but ceased due to the high cost and uncertainty of success. Engineered bacteriotherapies provide an alternative strategy for developing antimicrobials, with specific component parts (organism, enzyme, intended pathogen) that can be intentionally modified to address the challenges of particular infections. Though we present promising data for the ability to target $P$. aeruginosa PA14 biofilms using an engineered bacteriotherapy, further validation of this system is required in vivo. Expanding the pathogen targets, host infection sites, and adding additional functionalities, such as the production of specific antimicrobials, will better validate this system as an effective treatment alternative to existing therapies.

\section{METHODS}

Bacterial growth and transformation

All strains used in this study are listed in Supplementary Table 1. E. coli and $P$. aeruginosa were grown in LB broth and plated on LB agar, unless stated otherwise. Erythromycin and ampicillin were added to $E$. coli cultures at 200 or $100 \mu \mathrm{g} / \mathrm{mL}$, respectively. Lactobacilli were grown in De Man, Rogosa, and Sharpe (MRS; RPI Corp) broth and plated on MRS agar (1.5\% w/v) plates unless stated otherwise. Erythromycin was added to lactobacillus cultures at $5 \mu \mathrm{g} / \mathrm{mL}$ when necessary. All cultures were grown at $37^{\circ} \mathrm{C} ; \mathrm{E}$. coli and L. plantarum cultures were grown shaking (250 rpm) and LGG was grown statically, unless stated otherwise. E. coli transformation was performed using MES or TSS competent cells. L. plantarum WCFS1 transformation was performed using a method derived from Aukrust and Blom $^{67}$. LGG transformation was performed using the method described in De Keersmaecker et al. ${ }^{68}$.

\section{$\mathrm{LAB}$ antimicrobial plate assay}

Overnight cultures of $L A B$ were diluted $1000 \times$ into $10 \mathrm{~mL}$ fresh media and $1 \mathrm{~mL}$ aliquots were removed from the culture at the designated times. Following aliquot removal from LAB culture, cells were pelleted at $4000 \times g$ for $15 \mathrm{~min}$ and the resulting supernatant was filtered through $0.22 \mu \mathrm{m}$ PES filter and frozen at $-20^{\circ} \mathrm{C}$ until the following day. The following day, overnight cultures of $P$. aeruginosa were diluted $100 \times$ in LB broth and $100 \mu \mathrm{L}$ was plated on the surface of LB agar. Agar wells were excised from the agar plate and $200 \mu \mathrm{L}$ of fresh lactobacilli culture or filtered culture supernatant was added to each well. Plates were incubated at $37^{\circ} \mathrm{C}$ overnight and inhibition was evaluated qualitatively by inhibition of pathogen growth.

\section{Plasmid construction}

All vectors used and constructed in this study are listed in Supplementary Table 2. All DNA oligos were ordered from Eurofins Genomics or GENEWIZ and sequences are given in Supplementary Table 3. E. coli TG1 was modified by knockout of endA to improve transformation efficiency and plasmid quality of pSIP411-derived vectors. DH5a was used to propagate pSIP401-derived plasmids. Gene knockout and verification of endA in E. coli TG1 was performed using $\lambda$ Red recombineering ${ }^{69}$ using primers $17-20$. Variants of the pSIP401 and pSIP411 plasmids with inserts containing the Lp_3050 secretion signal, 6X histidine tag, thrombin cleavage site, and multiple cloning site (MCS) were constructed using primers 1-5 (Supplementary Table 3). The inserts for these constructs were generated by overlap extension PCR. The product and vectors (pSIP401 and pSIP411) were digested with Bg/ll and Pm/l to construct pTCC200 and pTCC210. The nucA gene was amplified from genomic DNA prepared from $S$. aureus UAMS1 using primers 6 and 7. The engZ gene was amplified from genomic DNA of C. cellulovorans (purchased from DSMZ) using primers 8 and 9. Amplified DNA fragments containing nucA or engZ were digested with Sall and Pm/l for insertion into the same digested pTCC200. The gene for PelA was amplified from genomic DNA prepared from PA14 using primers 10 and 11. Plasmid pTCC210 was amplified using primers 12 and 13 and combined with the Pel $A_{h}$ fragment using NEBuilder HiFi DNA Assembly. All enzymes were purchased from New England Biolabs (NEB, Ipswich, MA). All cloning inserts were amplified using Phusion DNA polymerase. All inserts in modified plasmids were verified by colony PCR and sequenced by Eurofins Genomics LLC (Louisville, KY) or Genewiz, Inc. (Cambridge, MA) using primers 14,15 , and 16 .

\section{Liquid culture biofilm formation}

$P$. aeruginosa PA14 biofilms were grown by diluting a $24 \mathrm{~h}$ culture $200 \mathrm{x}$ into salt-free LB (sfLB; $10 \mathrm{~g} / \mathrm{L}$ tryptone, $5 \mathrm{~g} / \mathrm{L}$ yeast extract). For biofilm inhibition studies, PA14 was also diluted $200 \times$ into the supernatants from $24 \mathrm{~h}$ cultures of $L$. plantarum and LGG, and this culture was subsequently serially diluted with a new sfLB PA14 subculture to maintain consistent cell density. The new PA14 cultures were dispensed in $150 \mu \mathrm{L}$ aliquots into wells of white Lumitrac high-bind 96-well microplates (Greiner Bio-One, Monroe, NC). Wells at the plate edge were filled with water and only interior wells were used for biofilm formation. Microplate lids were sealed with parafilm and incubated for $24 \mathrm{~h}$ at $30^{\circ} \mathrm{C}$ without shaking. Biofilms biomass was then quantified or incubated further to measure treatment efficacy.

\section{Biofilm quantification}

The biofilm biomass was measured by staining adherent cells with CV. Wash steps were performed using low pipette flow rates to prevent removal of adherent cells. Biofilms grown in liquid cultures as described above were washed $2 \times$ with $250 \mu \mathrm{L}$ PBS to remove non-adherent cells. $250 \mu \mathrm{L}$ of aqueous $0.1 \% \mathrm{CV}$ was added to each well, and plates were incubated for $15 \mathrm{~min}$. Following incubation, plates were inverted and washed $4 \times$ with $300 \mu \mathrm{L}$ phosphate-buffered saline (PBS; $8 \mathrm{~g} / \mathrm{L} \mathrm{NaCl}, 0.2 \mathrm{~g} / \mathrm{L}$

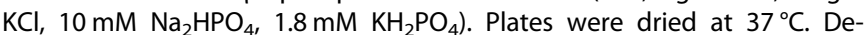
staining was performed by addition of $300 \mu \mathrm{L}$ of $4: 1$ ethanol:acetone solution. After $15-20 \mathrm{~min}, 200 \mu \mathrm{L}$ of the solubilized CV solution was transferred to a new 96-well microplate, and the absorbance at $570 \mathrm{~nm}$ wavelength was measured (Spectramax M3, Molecular Devices, San Jose, CA). 


\section{Enzyme induction and secretion validation}

Protein expression in L. plantarum and LGG was performed by growing overnight cultures in MRS and sub-culturing to $\mathrm{OD}_{600}$ of 0.05 in $\mathrm{BHI}$ supplemented with $0.5 \%(\mathrm{w} / \mathrm{v})$ glucose. Cultures were grown until $\mathrm{OD}_{600}$ $0.2-0.3$, pelleted, and induced by resuspending in fresh $2 \times \mathrm{BHI} 0.5 \%$ glucose with $200 \mathrm{ng} / \mathrm{mL} \mathrm{IP}-673$. Induced cultures were grown for $\sim 5 \mathrm{~h}$ at $30^{\circ} \mathrm{C}$. For induced supernatants, cells were pelleted by centrifugation at $4500 \times g$ for $10 \mathrm{~min}$, and the supernatant was filtered through a $0.22 \mu \mathrm{m}$ PES filter. Polymyxin B ( $100 \mu \mathrm{g} / \mathrm{mL}$, RPI Corp) was added to LAB cultures and supernatants when stated. For SDS-PAGE, $10 \mu \mathrm{L}$ of $4 \times$ loading buffer was added to $30 \mu \mathrm{L} \mathrm{LAB}$ supernatant and heated at $95^{\circ} \mathrm{C}$ for $5 \mathrm{~min} .20 \mu \mathrm{L}$ of the processed supernatant was loaded per well on a 4-12\% Bis-Tris gradient gel. The gel was run in $1 \times$ MES running buffer at $120 \mathrm{~V}$ for $\sim 1.5 \mathrm{~h}$ and developed using silver stain (Pierce Biotechnology Inc., Rockford, IL). Cellulase activity was evaluated by aliquoting $5 \mu \mathrm{L}$ of induced cell supernatants on $1.0 \%$ agar plates containing $0.5 \%$ carboxymethyl cellulose (CMC) and incubated overnight at $37^{\circ} \mathrm{C}$. Plates were subsequently incubated with $0.5 \%$ Congo Red (CR) for 10 min. Residual CR was removed by de-staining with $1 \mathrm{M} \mathrm{NaCl}$. DNase activity was assessed by aliquoting $5 \mu \mathrm{L}$ of induced cell supernatants on $1.0 \%$ agar plates containing $0.2 \%$ DNA and incubated overnight at $37^{\circ} \mathrm{C}$. Plates were then treated with $1 \mathrm{~N} \mathrm{HCl}$ to precipitate residual DNA.

\section{Enzymatic degradation of PA14 biofilms by engineered LAB}

Efficacy of enzymatic treatment was determined by growing biofilms as described above. The supernatant and nonadherent solids of biofilm cultures were aspirated using a multichannel pipette. $250 \mu \mathrm{L}$ of induced $\mathrm{LAB}$ culture was aliquoted per well, and biofilm microplates were placed on a rocker at room temperature for $15 \mathrm{~h}$. LAB cultures were aspirated from wells and the plates were washed twice with $250 \mu \mathrm{L}$ of sterile DPBS

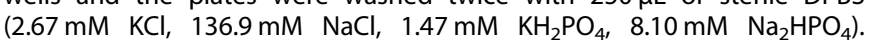
Remaining biofilm was fixed to plates by drying plates overnight in a $37^{\circ} \mathrm{C}$ incubator and quantified using the CV method described previously.

\section{Biofilm viability assay}

PA14 biofilms were grown as described above. Filtered LAB supernatants from $24 \mathrm{~h}$ LAB cultures were generated as described in the agar-well diffusion assay, and then serially diluted $2 x$ into PBS pH 7.4. The supernatant from the biofilm cultures was removed and $250 \mu \mathrm{L}$ of diluted LAB supernatants or buffered MRS control was added to each well and the plates were incubated at $37^{\circ} \mathrm{C}$ for $24 \mathrm{~h}$. The supernatant was then removed and the plates were washed $2 \times$ with PBS to remove nonadherent cells. $200 \mu \mathrm{L}$ of $\mathrm{LB}$ and $100 \mu \mathrm{L}$ of $\mathrm{XTT}$ solution $(0.4 \mathrm{mg} / \mathrm{mL} \mathrm{XTT}$, Amresco; $50 \mu \mathrm{M}$ menadione, Alfa Aesar) were added to each well and plates were incubated at $37^{\circ} \mathrm{C}$ for $3 \mathrm{~h}$. Microplates were centrifuged at $3000 \times g$ for $10 \mathrm{~min}$ and $200 \mu \mathrm{L}$ of solution was aliquoted into a fresh microplate. The absorbance at $475 \mathrm{~nm}$ was taken to determine viability and this value is reported in the corresponding figures.

\section{Reporting summary}

Further information on research design is available in the Nature Research Reporting Summary linked to this article.

\section{DATA AVAILABILITY}

The data that support the findings of this study are available from the corresponding author upon reasonable request.

Received: 8 April 2020; Accepted: 7 October 2020; Published online: 30 October 2020

\section{REFERENCES}

1. Wu, Y. K., Cheng, N. C. \& Cheng, C. M. Biofilms in chronic wounds: pathogenesis and diagnosis. Trends Biotechnol. https://doi.org/10.1016/j.tibtech.2018.10.011 (2019).

2. Delcaru, C. et al. Microbial biofilms in urinary tract infections and prostatitis: etiology, pathogenicity, and combating strategies. Pathogens https://doi.org/ 10.3390/pathogens5040065 (2016).
3. Hall-Stoodley, L., Costerton, J. W. \& Stoodley, P. Bacterial biofilms: from the natural environment to infectious diseases. Nat. Rev. Microbiol. https://doi.org/ 10.1038/nrmicro821 (2004).

4. von Rosenvinge, E. C., O'May, G. A., Macfarlane, S., Macfarlane, G. T. \& Shirtliff, M. E. Microbial biofilms and gastrointestinal diseases. Pathog. Dis. https://doi.org/ 10.1111/2049-632X.12020 (2013).

5. Kavanagh, N. et al. Staphylococcal osteomyelitis: disease progression, treatment challenges, and future directions. Clin. Microbiol. Rev. https://doi.org/10.1128/ CMR.00084-17 (2018).

6. Bispo, P. J. M., Haas, W. \& Gilmore, M. S. Biofilms in infections of the eye. Pathogens https://doi.org/10.3390/pathogens4010111 (2015).

7. Schaudinn, C., Gorur, A., Keller, D., Sedghizadeh, P. P. \& Costerton, J. W. Periodontitis: an archetypical biofilm disease. J. Am. Dent. Assoc. https://doi.org/ 10.14219/jada.archive.2009.0307 (2009).

8. Flemming, H.-C. \& Wingender, J. The biofilm matrix. Nat. Rev. Microbiol. 8, 623-633 (2010).

9. Gilbert, P., Maira-Litran, T., McBain, A. J., Rickard, A. H. \& Whyte, F. W. The physiology and collective recalcitrance of microbial biofilm communities. Adv. Microb. Physiol. https://doi.org/10.1016/S0065-2911(02)46005-5 (2002).

10. Domenech, M., Ramos-Sevillano, E., García, E., Moscoso, M. \& Yuste, J. Biofilm formation avoids complement immunity and phagocytosis of Streptococcus pneumoniae. Infect. Immun. https://doi.org/10.1128/IAl.00491-13 (2013).

11. Toussaint, J. et al. Topical antibiotic ointment versus silver-containing foam dressing for second-degree burns in swine. Acad. Emerg. Med. https://doi.org/ 10.1111/acem.12723 (2015).

12. Innes, M. E., Umraw, N., Fish, J. S., Gomez, M. \& Cartotto, R. C. The use of silver coated dressings on donor site wounds: a prospective, controlled matched pair study. Burns https://doi.org/10.1016/S0305-4179(01)00015-8 (2001).

13. Poon, V. K. M. \& Burd, A. In vitro cytotoxity of silver: implication for clinical wound care. Burns https://doi.org/10.1016/j.burns.2003.09.030 (2004).

14. Michaels, J. A. et al. Randomized controlled trial and cost-effectiveness analysis of silver-donating antimicrobial dressings for venous leg ulcers (VULCAN trial). Br. J. Surg. https://doi.org/10.1002/bjs.6786 (2009).

15. Pestrak, M. J. et al. Treatment with the pseudomonas aeruginosa glycoside hydrolase PsIG combats wound infection by improving antibiotic efficacy and host innate immune activity. Antimicrob. Agents Chemother. https://doi.org/ 10.1128/AAC.00234-19 (2019).

16. Baker, P. et al. Exopolysaccharide biosynthetic glycoside hydrolases can be utilized to disrupt and prevent Pseudomonas aeruginosa biofilms. Sci. Adv. 2, e1501632 (2016).

17. Frederiksen, B., Pressler, T., Hansen, A., Koch, C. \& Høiby, N. Effect of aerosolized rhDNase (Pulmozyme) on pulmonary colonization in patients with cystic fibrosis. Acta Paediatr. 95, 1070-1074 (2006).

18. Johansen, C., Falholt, P. \& Gram, L. Enzymatic removal and disinfection of bacterial biofilms. Appl. Environ. Microbiol. 63, 3724-3728 (1997).

19. Lu, T. K. \& Collins, J. J. Dispersing biofilms with engineered enzymatic bacteriophage. Proc. Natl Acad. Sci. USA 104, 11197-11202 (2007).

20. Lister, J. L. \& Horswill, A. R. Staphylococcus aureus biofilms: recent developments in biofilm dispersal. Front. Cell. Infect. Microbiol. https://doi.org/10.3389/ fcimb.2014.00178 (2014).

21. Fleming, D. \& Rumbaugh, K. Approaches to dispersing medical biofilms. Microorganisms https://doi.org/10.3390/microorganisms5020015 (2017).

22. Bober, J. R., Beisel, C. L. \& Nair, N. U. Synthetic biology approaches to engineer probiotics and members of the human microbiota for biomedical applications. Annu. Rev. Biomed. Eng. https://doi.org/10.1146/annurev-bioeng-062117-121019 (2018).

23. Riglar, D. T. \& Silver, P. A. Engineering bacteria for diagnostic and therapeutic applications. Nat. Rev. Microbiol. https://doi.org/10.1038/nrmicro.2017.172 (2018).

24. Mays, Z. J. \& Nair, N. U. Synthetic biology in probiotic lactic acid bacteria: at the frontier of living therapeutics. Curr. Opin. Biotechnol. https://doi.org/10.1016/j. copbio.2018.01.028 (2018).

25. Waller, M. C., Bober, J. R., Nair, N. U. \& Beisel, C. L. Toward a genetic tool development pipeline for host-associated bacteria. Curr. Opin. Microbiol. https://doi. org/10.1016/j.mib.2017.05.006 (2017).

26. Martín, R. et al. Effects in the use of a genetically engineered strain of Lactococcus lactis delivering in situ IL-10 as a therapy to treat low-grade colon inflammation. Hum. Vaccines Immunother. https://doi.org/10.4161/hv.28549 (2014).

27. Hwang, I. Y. et al. Engineered probiotic Escherichia coli can eliminate and prevent Pseudomonas aeruginosa gut infection in animal models. Nat. Commun. https:// doi.org/10.1038/ncomms15028 (2017).

28. Isabella, V. M. et al. Development of a synthetic live bacterial therapeutic for the human metabolic disease phenylketonuria. Nat. Biotechnol. https://doi.org/ 10.1038/nbt.4222 (2018). 
29. Gurbatri, C. R. et al. Engineered probiotics for local tumor delivery of checkpoint blockade nanobodies. Sci. Transl. Med. https://doi.org/10.1126/scitranslmed. aax0876 (2020).

30. Han, W. et al. Improvement of an experimental colitis in rats by lactic acid bacteria producing superoxide dismutase. Inflamm. Bowel Dis. https://doi.org/ 10.1097/01.mib.0000235101.09231.9e (2006).

31. del Carmen, S. et al. Genetically engineered immunomodulatory Streptococcus thermophilus strains producing antioxidant enzymes exhibit enhanced anti-inflammatory activities. Appl. Environ. Microbiol. https://doi.org/10.1128/ AEM.03296-13 (2014).

32. Vedantam, G. et al. An engineered synthetic biologic protects against Clostridium difficile infection. Front. Microbiol. https://doi.org/10.3389/fmicb.2018.02080 (2018).

33. Jamalifar, H. et al. Antimicrobial activity of different Lactobacillus species against multi-drug resistant clinical isolates of Pseudomonas aeruginosa. Iran. J. Microbiol. 3, 21-25 (2011).

34. Peral, M. C., Huaman Martinez, M. A. \& Valdez, J. C. Bacteriotherapy with Lactobacillus plantarum in burns. Int. Wound J. 6, 73-81 (2009).

35. Khailova, L., Baird, C. H., Rush, A. A., McNamee, E. N. \& Wischmeyer, P. E. Lactobacillus rhamnosus GG improves outcome in experimental Pseudomonas aeruginosa pneumonia: potential role of regulatory T cells. Shock https://doi.org/ 10.1097/SHK.0000000000000066 (2013).

36. Mohammedsaeed, W., Cruickshank, S., McBain, A. J. \& O'Neill, C. A. Lactobacillus rhamnosus GG lysate increases re-epithelialization of keratinocyte scratch assays by promoting migration. Sci. Rep. https://doi.org/10.1038/srep16147 (2015).

37. Argenta, A., Satish, L., Gallo, P., Liu, F. \& Kathju, S. Local application of probiotic bacteria prophylaxes against sepsis and death resulting from burn wound infection. PLoS ONE 11, e0165294 (2016).

38. Sabaeifard, P., Abdi-Ali, A., Soudi, M. R. \& Dinarvand, R. Optimization of tetrazolium salt assay for Pseudomonas aeruginosa biofilm using microtiter plate method. J. Microbiol. Methods https://doi.org/10.1016/j.mimet.2014.07.024 (2014).

39. Branda, S. S., Vik, S., Friedman, L. \& Kolter, R. Biofilms: the matrix revisited. Trends Microbiol. 13, 20-26 (2005).

40. Lee, D. G. et al. Genomic analysis reveals that Pseudomonas aeruginosa virulence is combinatorial. Genome Biol. 7, R90 (2006).

41. Wozniak, D. J. et al. Alginate is not a significant component of the extracellular polysaccharide matrix of PA14 and PAO1 Pseudomonas aeruginosa biofilms. Proc. Natl Acad. Sci. USA 100, 7907-7912 (2003).

42. Jennings, L. K. et al. Pel is a cationic exopolysaccharide that cross-links extracellular DNA in the Pseudomonas aeruginosa biofilm matrix. Proc. Natl Acad. Sci. USA 112, 11353-11358 (2015).

43. Whitchurch, C. B., Tolker-Nielsen, T., Ragas, P. C. \& Mattick, J. S. Extracellular DNA required for bacterial biofilm formation. Science (80-.). 295, 1487-1487 (2002).

44. Friedman, L. \& Kolter, R. Genes involved in matrix formation in Pseudomonas aeruginosa PA14 biofilms. Mol. Microbiol. 51, 675-690 (2004).

45. Jeon, S. D., Yu, K. O., Kim, S. W. \& Han, S. O. The processive endoglucanase EngZ is active in crystalline cellulose degradation as a cellulosomal subunit of Clostridium cellulovorans. New Biotechnol. 29, 365-371 (2012).

46. Cuatrecasas, P., Fuchs, S. \& Anfinsen, C. B. Catalytic properties and specificity of the extracellular nuclease of Staphylococcus aureus. J. Biol. Chem. 242, 1541-1547 (1967).

47. Anderssen, E. L., Diep, D. B., Nes, I. F., Eijsink, V. G. H. \& Nissen-Meyer, J. Antag onistic activity of Lactobacillus plantarum C11: two new two-peptide bacteriocins, plantaricins $\mathrm{EF}$ and $\mathrm{JK}$, and the induction factor plantaricin A. Appl. Environ. Microbiol. https://doi.org/10.1139/m93-178 (1998).

48. Diep, D. B., Straume, D., Kjos, M., Torres, C. \& Nes, I. F. An overview of the mosaic bacteriocin pln loci from Lactobacillus plantarum. Peptides https://doi.org/ 10.1016/j.peptides.2009.05.014 (2009).

49. Lu, R. et al. Isolation, identification, and characterization of small bioactive peptides from Lactobacillus GG conditional media that exert both anti-Gram-negative and Gram-positive bactericidal activity. J. Pediatr. Gastroenterol. Nutr. https://doi. org/10.1097/MPG.0b013e3181924d1e (2009).

50. Zalán, Z., Hudáček, J., Štětina, J., Chumchalová, J. \& Halász, A. Production of organic acids by Lactobacillus strains in three different media. Eur. Food Res. Technol. https://doi.org/10.1007/s00217-009-1179-9 (2010).

51. Silva, M., Jacobus, N. V., Deneke, C. \& Gorbach, S. L. Antimicrobial substance from a human Lactobacillus strain. Antimicrob. Agents Chemother. https://doi.org/ 10.1128/AAC.31.8.1231 (1987).

52. Alakomi, H. L. et al. Lactic acid permeabilizes Gram-negative bacteria by disrupting the outer membrane. Appl. Environ. Microbiol. https://doi.org/10.1128/ AEM.66.5.2001-2005.2000 (2000).

53. Ryssel, $H$. et al. The antimicrobial effect of acetic acid-an alternative to common local antiseptics? Burns https://doi.org/10.1016/j.burns.2008.11.009 (2009).

54. De Keersmaecker, S. C. J. et al. Strong antimicrobial activity of Lactobacillus rhamnosus GG against Salmonella typhimurium is due to accumulation of lactic acid. FEMS Microbiol. Lett. https://doi.org/10.1111/j.1574-6968.2006.00250.x (2006).

55. Ratzke, C. \& Gore, J. Modifying and reacting to the environmental pH can drive bacterial interactions. PLoS Biol. https://doi.org/10.1371/journal.pbio.2004248 (2018)

56. Valdéz, J. C., Peral, M. C., Rachid, M., Santana, M. \& Perdigón, G. Interference of Lactobacillus plantarum with Pseudomonas aeruginosa in vitro and in infected burns: the potential use of probiotics in wound treatment. Clin. Microbiol. Infect. 11, 472-479 (2005)

57. Gethin, G. The significance of surface $\mathrm{pH}$ in chronic wounds. Wounds UK 3, 52-56 (2007).

58. Greener, B., Hughes, A. A., Bannister, N. P. \& Douglass, J. Proteases and pH in chronic wounds. J. Wound Care https://doi.org/10.12968/jowc.2005.14.2.26739 (2005).

59. Nagoba, B. S., Suryawanshi, N. M., Wadher, B. \& Selkar, S. Acidic environment and wound healing: a review. Wounds 27, 5-11 (2015).

60. Lebeer, S., Verhoeven, T. L. A., Vélez, M. P., Vanderleyden, J. \& De Keersmaecker, S. C. J. Impact of environmental and genetic factors on biofilm formation by the probiotic strain Lactobacillus rhamnosus GG. Appl. Environ. Microbiol. https://doi. org/10.1128/AEM.01393-07 (2007).

61. Allesen-Holm, M. et al. A characterization of DNA release in Pseudomonas aeruginosa cultures and biofilms. Mol. Microbiol. https://doi.org/10.1111/j.13652958.2005.05008.x (2006).

62. Mulcahy, H., Charron-Mazenod, L. \& Lewenza, S. Extracellular DNA chelates cations and induces antibiotic resistance in Pseudomonas aeruginosa biofilms. PLoS Pathog. https://doi.org/10.1371/journal.ppat.1000213 (2008).

63. Chiang, W. C. et al. Extracellular DNA shields against aminoglycosides in Pseudomonas aeruginosa biofilms. Antimicrob. Agents Chemother. https://doi.org/ 10.1128/AAC.00001-13 (2013).

64. Fuxman Bass, J. I. et al. Extracellular DNA: a major proinflammatory component of Pseudomonas aeruginosa biofilms. J. Immunol. https://doi.org/10.4049/ jimmunol.0901640 (2010).

65. Das, T. et al. Phenazine virulence factor binding to extracellular DNA is important for Pseudomonas aeruginosa biofilm formation. Sci. Rep. https://doi.org/10.1038/ srep08398 (2015).

66. Kim, S. Y., Park, S. Y., Choi, S. K. \& Park, S. H. Biosynthesis of polymyxins B, E, and P using genetically engineered polymyxin synthetases in the surrogate host Bacillus subtilis. J. Microbiol. Biotechnol. https://doi.org/10.4014/jmb.1505.05036 (2015).

67. Aukrust, T. \& Blom, H. Transformation of Lactobacillus strains used in meat and vegetable fermentations. Food Res. Int. 25, 253-261 (1992).

68. De Keersmaecker, S. C. J. et al. Flow cytometric testing of green fluorescent protein-tagged Lactobacillus rhamnosus GG for response to defensins. Appl. Environ. Microbiol. https://doi.org/10.1128/AEM.02605-05 (2006).

69. Datsenko, K.A. \& Wanner, B. L. One-step inactivation of chromosomal genes in Escherichia coli K-12 using PCR products. Proc. Natl Acad. Sci. USA 97, 6640-6645 (2000).

\section{ACKNOWLEDGEMENTS}

We would like to thank Dr. David R. Snydman (Tufts Medical Center, Boston, MA), Dr Abraham L. Sonenshein (Tufts University School of Medicine, Boston, MA), Dr. Michiel Kleerebezem (Wageningen University \& Research, Wageningen, Netherlands), Dr. Roberto Kolter (Harvard Medical School, Boston, MA), Dr. Ann Hochschild (Harvard Medical School, Boston, MA), Dr. Huimin Zhao (University of Illinois, Urbana, IL), Dr. Geir Mathiesen (Norwegian University of Life Sciences, Ås, Norway), and Dr. Jan Peter van Pijkeren (University of Wisconsin, Madison, WI) for graciously providing strains and/or plasmids required to complete this work. This work was financially supported by grant number DP2HD091798 of the National Institutes of Health and a Tufts Collaborates! grant from Tufts University.

\section{AUTHOR CONTRIBUTIONS}

Project was conceived by N.U.N. and experiments were designed by N.U.N. and T.C.C. Laboratory work and data analysis was performed by T.C.C. Manuscript was written by T.C.C. and edited by N.U.N.

\section{COMPETING INTERESTS}

The authors declare no competing interests. 


\section{ADDITIONAL INFORMATION}

Supplementary information is available for this paper at https://doi.org/10.1038/ s41522-020-00156-6.

Correspondence and requests for materials should be addressed to N.U.N.

Reprints and permission information is available at http://www.nature.com/ reprints

Publisher's note Springer Nature remains neutral with regard to jurisdictional claims in published maps and institutional affiliations.
Open Access This article is licensed under a Creative Commons Attribution 4.0 International License, which permits use, sharing, adaptation, distribution and reproduction in any medium or format, as long as you give appropriate credit to the original author(s) and the source, provide a link to the Creative Commons license, and indicate if changes were made. The images or other third party material in this article are included in the article's Creative Commons license, unless indicated otherwise in a credit line to the material. If material is not included in the article's Creative Commons license and your intended use is not permitted by statutory regulation or exceeds the permitted use, you will need to obtain permission directly from the copyright holder. To view a copy of this license, visit http://creativecommons. org/licenses/by/4.0/.

(c) The Author(s) 2020 